\title{
Development of Mold for Demolding Resistance Measurement in Polymer Injection Molding
}

\author{
Yasuhiko Murata, ${ }^{1 *}$ Takahiro Nakanishi, ${ }^{1}$ Naoki Hirai, ${ }^{1}$ \\ Fumiya Kawanishi, ${ }^{1}$ and Hidehiko Ibuki ${ }^{2}$ \\ ${ }^{1}$ Nippon Institute of Technology, \\ 4-1 Gakuendai, Miyashiro, Minamisaitama, Saitama 345-8501, Japan \\ ${ }^{2}$ Osaka Gas Chemicals, Ltd., 5-11-61, Torishima, Konohana, Osaka 554-0051, Japan
}

(Received March 4, 2019; accepted May 7, 2019)

Keywords: polymer lens, injection molding, demolding resistance, quartz force sensor

In the injection molding of polymer lenses, the demolding resistance may cause problems, such as deformation, cracks, and scratches, in these products. To examine the method of decreasing the demolding resistance of polymer lenses, a method of precisely measuring the demolding resistance should be established. In this study, we designed and fabricated a mold equipped with high-sensitivity quartz force sensors for demolding resistance measurement that is customized for the injection molding of polymer lenses. In addition, we evaluated the performance of the mold. Using this mold, we measured the demolding resistance of fluorine-based polyester lenses. As a result, it was clarified that (1) the preload applied to the quartz force sensors before the start of mold closing has a significant effect on the generation of demolding resistance, (2) the draft angle of a cavity wall has an effect on the demolding resistance, and (3) the maximum demolding resistance increases with increasing holding pressure, injection rate, heating cylinder temperature, and mold temperature.

\section{Introduction}

Recently, polymers have been widely used as the material of optical lenses. Most polymer lenses are fabricated by injection molding. A high precision of the shape, dimensions, and surface properties is required for optical lenses. To satisfy these requirements, a high pressure must be applied to the polymer injected into a mold during injection molding to achieve close contact between the polymer and the surface of the mold cavity so that the shape and surface properties of the cavity are sufficiently transcribed onto the lens surface. However, when the shape and surface properties of the cavity are sufficiently transcribed, it is difficult to demold molded products from the cavity surface because the products are securely attached to the cavity surface. This is a problem during the demolding process. The level of this difficulty is called the demolding resistance. As we try to improve the precision of the shape, dimensions, and surface properties of the molded products by increasing the transcription quality, the

*Corresponding author: e-mail: ymurata@nit.ac.jp

https://doi.org/10.18494/SAM.2019.2357 
demolding resistance increases. As a result, product defects, such as deformation, cracks, and scratches, are generated during the demolding process. Therefore, the method of decreasing the demolding resistance while maintaining a high level of transcription has been examined. One example is the examination of a mold release agent added to the molding material. In addition, coating the cavity surface with diamond-like carbon with a mold release characteristic ${ }^{(1)}$ or with an organic-based material, ${ }^{(2,3)}$ as well as adjusting the cavity surface by shot blasting so that demolding becomes easier ${ }^{(4,5)}$ have been examined. As explained, various methods of decreasing the demolding resistance have been actively examined. However, it has been difficult to evaluate the effects of these methods because no method of precisely measuring the demolding resistance for molded lenses has been established thus far. For molded products other than lenses, various molds for demolding resistance measurement have been proposed and used in experiments. For example, methods of measuring the demolding resistance on (1) the inner surface of molded products with a cylindrical or cup shape, (2) the sidewall of ribs, and (3) the cavity surface with microstructures, including lines and spaces, using load cells have been proposed. ${ }^{(6-10)}$ However, in those methods, the demolding resistance generated along the surface to which the molded product is attached is the measurement target. In this case, the demolding resistance is large and is easily measured because the demolding resistance is mainly caused by the friction resistance and the anchor effect between the molded product and the cavity surface. In contrast, for lenses, the surface properties are good and the demolding resistance is generated in the direction perpendicular to the surface on which the molded lens is attached. In this case, the demolding resistance is caused by surface tension and is relatively small, making the measurement extremely difficult. Under these conditions, a mold that is equipped with a high-sensitivity quartz force sensor for demolding resistance measurement is required to measure the small demolding resistance of lenses fabricated under various conditions of cavity shape, surface properties, and surface treatment.

In this study, we designed and fabricated a new mold for the demolding resistance measurement of polymer lenses that satisfies the above requirements. We first evaluated the performance of the mold and experimentally demonstrated its advantages by measuring the demolding resistance under various molding conditions.

\section{Mold for Demolding Resistance Measurement}

\subsection{Measurement principle of demolding resistance}

Figure 1 shows the measurement principle of demolding resistance. During the injection molding process, a molded product attaches to the surface of a cylindrical cavity insert set into the cavity. When ejector pins are pushed forward by the protruding equipment of the injection molding machine, the molded product is ejected from the cavity. Upon this ejection, the cavity insert as well as the molded product are pulled in the ejection direction. This pulling force corresponds to the demolding resistance and is measured using the quartz force sensor. 


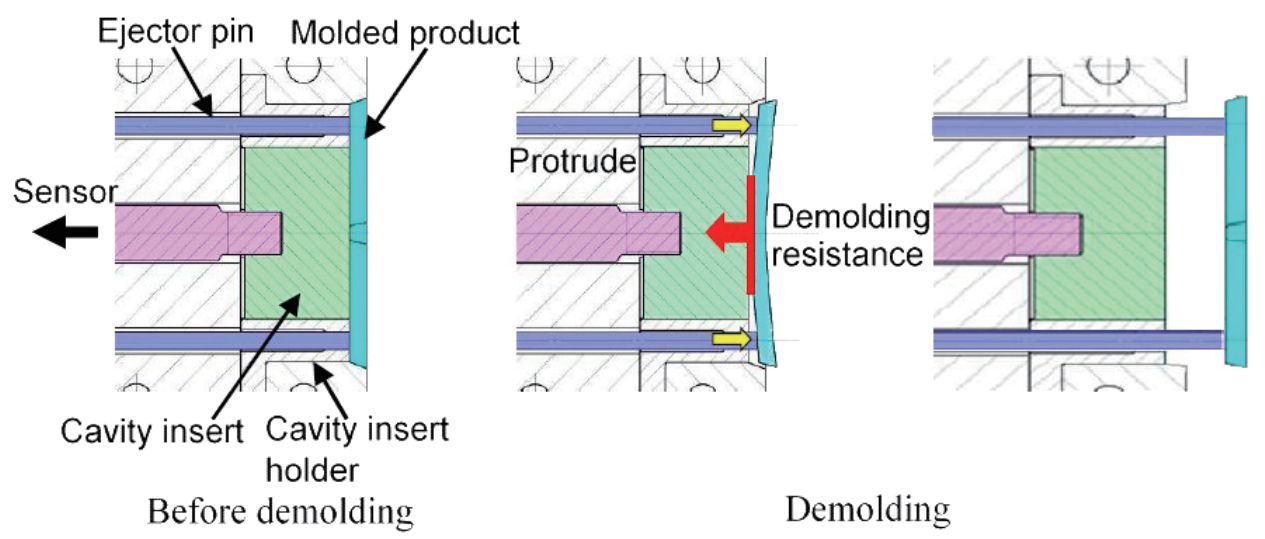

Fig. 1. (Color online) Measurement principle of demolding resistance.

\subsection{Basic structure of mold}

Figure 2 shows the basic structure of the mold for demolding resistance measurement designed and fabricated in this study. Two cavities are positioned symmetrically with respect to the central axis of the mold. This mold includes a demolding resistance measurement unit equipped with a quartz force sensor. In addition, inductive displacement sensors (EX$110 \mathrm{~V}$, Keyence Corporation) are attached to the upper and lower surfaces of an ejector plate to measure the forward motion of ejector pins. The changes in demolding resistance and protruded displacement with time were simultaneously recorded. Figure 3 shows an enlarged view of the unit for demolding resistance measurement. As explained in Sect. 1, the demolding resistance generated in polymer lenses is small. Therefore, the measurement sensitivity of conventional force sensors is considered to be too low to obtain a sufficient measurement accuracy. In this study, we used a piezoelectric quartz force sensor (Type 9217A, Kistler) that can measure a small force of less than $500 \mathrm{~N}$ with high sensitivity. Table 1 shows a summary of the performance characteristics of three quartz force sensors: two types of piezoelectric quartz force sensor (Type $9173 \mathrm{~B}^{(6)}$ and Type $9301 \mathrm{~B},{ }^{(10)}$ Kistler) that have been conventionally used in studies targeting the measurement of demolding resistance generated along the surface to which a molded product is attached and the high-sensitivity quartz force sensor used in this study. The sensitivity of the high-sensitivity quartz force sensor is 10 - to 25 -fold higher than that of the former two force sensors. Therefore, it is possible to accurately measure a very small demolding resistance generated orthogonally to the surface to which the molded product is attached. In addition, its applicable temperature range is the widest among the three sensors, making it suitable for the measurement of demolding resistance generated during lens molding, which requires a mold temperature higher than $100^{\circ} \mathrm{C}$.

The sensor used in this study is suitable for measuring a compressive force owing to its structure. Sensor supports A and B are connected in a chainlike manner and the sensor is placed between them so that the demolding resistance applied in the tensile direction is measured after converting it to the compressive force. Sensor support A is pulled towards the 


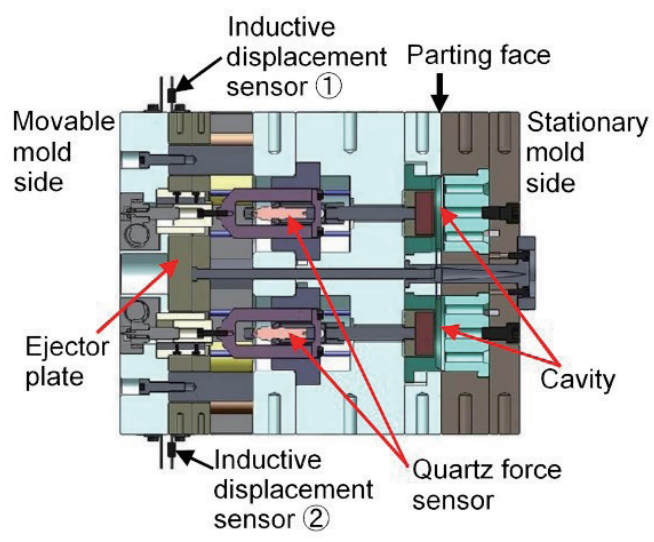

Fig. 2. (Color online) Structure of demolding resistance measurement mold.

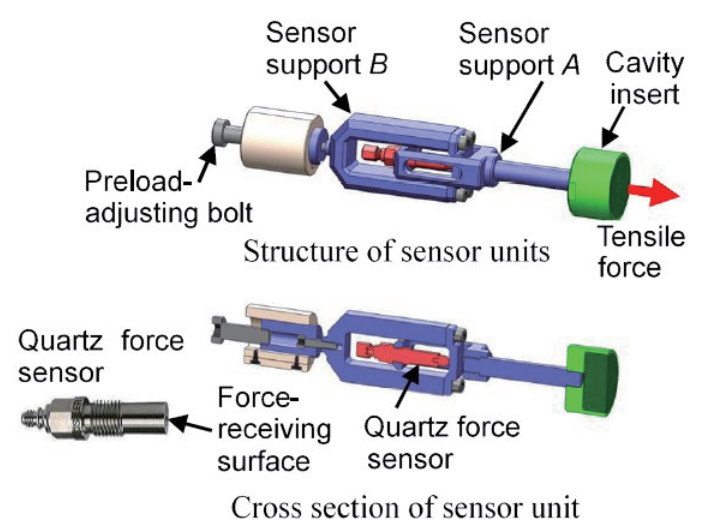

Fig. 3. (Color online) Structure of demolding resistance measurement unit.

Table 1

Comparison of sensor characteristics.

\begin{tabular}{lccc}
\hline Sensor characteristic & $\begin{array}{c}\text { Quartz force } \\
\text { sensor } \\
\text { Type 9173B }\end{array}$ & $\begin{array}{c}\text { Quartz force } \\
\text { sensor } \\
\text { Type 9301B }\end{array}$ & $\begin{array}{c}\text { High-sensitivity } \\
\text { quartz force sensor } \\
\text { Type 9217A }\end{array}$ \\
\hline Measurement range (N) & $0-12000$ & \pm 2500 & \pm 500 \\
Sensitivity (pc/N) & -3.5 & -4 & -105 \\
Operating temperature range $\left({ }^{\circ} \mathrm{C}\right)$ & $-20-80$ & $-40-120$ & $-80-205$ \\
\hline
\end{tabular}

right in Fig. 3, upon the ejection of the molded product since the molded product is attached to the cavity insert. Because of this, the force-receiving surface of the sensor is pushed onto the surface of sensor support B. This pushing force corresponds to the demolding resistance. When there is a gap between the force-receiving surface of the sensor and sensor support B, correct measurement is impossible. To avoid the existence of such gaps, or to realize the state that force is constantly applied to the sensor, a preload-adjusting device (explained later) is installed in the mold.

Figure 4 shows the shape of the cavity. In this study, to simplify the evaluation of the mold, the cavity surface was not curved, unlike the surface of the lens; instead, it was a circular plate of $60 \mathrm{~mm}$ diameter and $4 \mathrm{~mm}$ thickness. A side-gate structure was adopted. The surface of the $40 \mathrm{~mm}$ diameter cavity insert, instead of the entire cavity surface, was the measurement target. The surface roughness $(R a)$ of the inserts of the upper and lower cavities were 0.023 and $0.029 \mu \mathrm{m}$, respectively. The draft angle of the cavity wall was changed $\left(5,10,15\right.$, or $\left.30^{\circ}\right)$ to examine its effect on the demolding resistance. An undercut part was formed at the tip of each of the four ejector pins used to eject the molded product from the cavity so that the molded product was kept on the movable side of the cavity upon mold opening. 


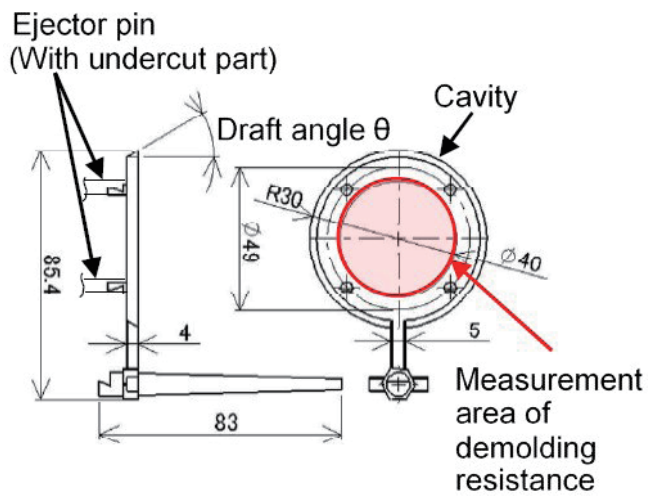

Fig. 4. (Color online) Shape of molded product (units: $\mathrm{mm})$.

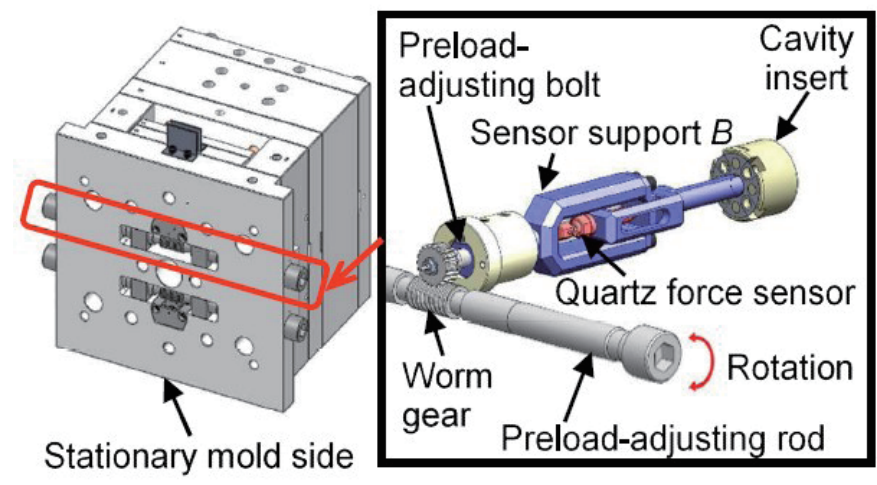

Fig. 5. (Color online) Structure of preload-adjusting device.

\subsection{Preload-adjusting device}

During the molding process, the thickness of the mold changes depending on the mold clamping force and the shrinkage and expansion of the mold plate caused by the change in mold temperature. When the thickness of the mold changes during measurement, the relative positions of sensor supports A and B change, causing a change in the preload set beforehand and hence a variation in measurement value. Therefore, a means to adjust the preload during the molding process is necessary. The preload-adjusting device shown in Fig. 5 was placed inside the mold to adjust the preload from the operation panel of the injection molding machine, while the mold was placed at the platen of the machine. A worm gear was attached to the preloadadjusting rod connected to sensor support B. By rotating the preload-adjusting rod, the preloadadjusting bolt connected to the worm gear was rotated, enabling the adjustment of the preload from the side of the mold.

\section{Calibration of Output of Quartz Force Sensor}

A calibration experiment of the output was carried out using the mold. Figure 6 shows the experimental apparatus used for output calibration. A load was applied to the cavity insert using weights while changing the preload applied to the quartz force sensor in a stepwise manner from 0 to $30 \mathrm{~N}$ to measure the output. Figure 7 shows the relationship between the output (sensor output - preload) and the weight for different preloads. The dotted line indicates the ideal line assuming no frictional resistance at the sliding surface between the cavity insert and the mold base. For the preload of 0 to $10 \mathrm{~N}$, the output linearly increases with increasing weight. For the preload of more than $15 \mathrm{~N}$, the output curve gradually deviates from the ideal line and loses linearity. Before the calibration experiment, a linear relationship was expected between the output and the weight even with changing preload. However, the preload was found to significantly affect the sensor output. The reason behind this is the internal structure 


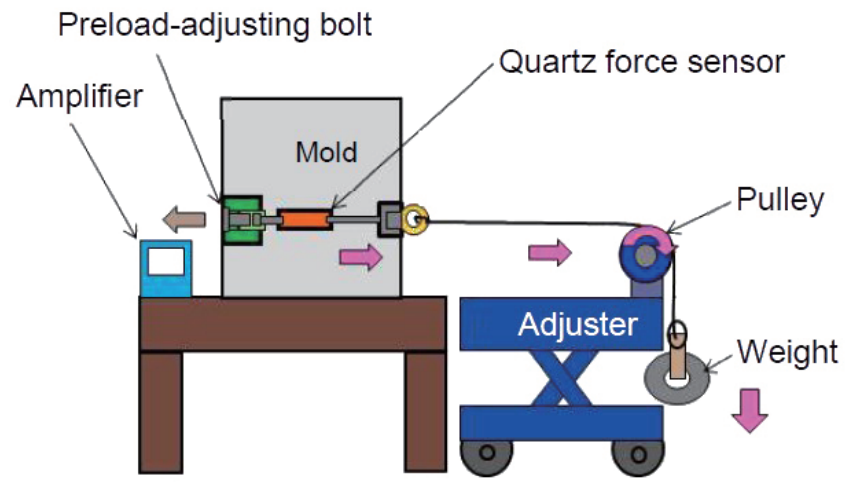

Fig. 6. (Color online) Experimental apparatus for calibration.

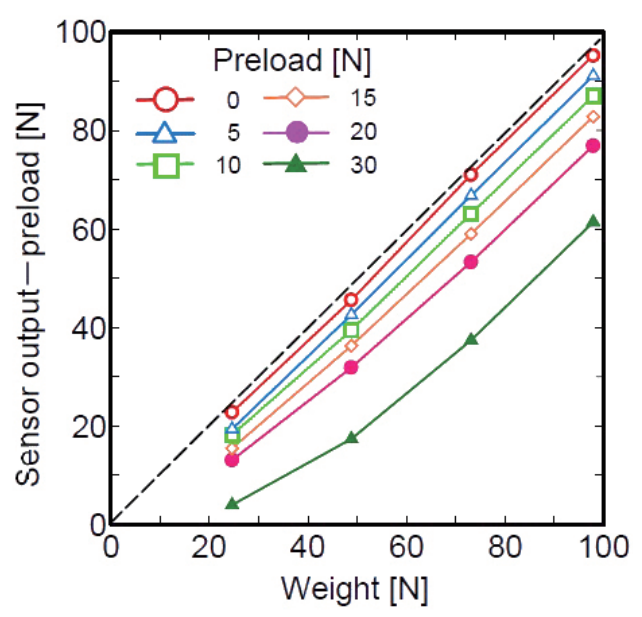

Fig. 7. (Color online) Result of calibration.

of the quartz force sensor. On the basis of the above findings, the preload should be set at less than $10 \mathrm{~N}$ during measurement to obtain a curve that approximates the ideal line.

\section{Experimental Method}

The mold developed in this study was attached to the injection molding machine (ROBOSHOT $\alpha$-50iAP, Fanuc Corporation; maximum mold clamping force, $490 \mathrm{kN}$; screw diameter, $26 \mathrm{~mm}$ ) to measure the demolding resistance. For the polymer, the fluorine-based polyester generally used for polymer lenses (FBP2-2, Osaka Gas Chemicals, Ltd.) was used. Table 2 shows the molding conditions. In this study, the demolding resistance was measured by changing the holding pressure, injection rate, heating cylinder temperature, and mold temperature. The values underlined in Table 2 were the reference conditions. Considering the findings obtained in Sect. 3, the preload was set to $10 \mathrm{~N}$ before each mold closing. After the molding process, the demolding resistance was measured five times and the value closest to the average of the five measurements was adopted.

\section{Effect of Draft Angle of Cavity Wall on Demolding Resistance}

\subsection{Sensor output during mold opening}

Figure 8 shows the change in sensor output with time for different draft angles of the cavity wall from the start of mold opening to the completion of molded product ejection using ejector pins. The sensor output measured at the upper cavity was obtained when the holding pressure was $60 \mathrm{MPa}$. At a cavity draft angle of less than $15^{\circ}$, the sensor output decreased to $0 \mathrm{~N}$ before 
Table 2

Molding conditions.

\begin{tabular}{lc}
\hline Heating cylinder temperature ${ }^{*}\left({ }^{\circ} \mathrm{C}\right)$ & $\underline{262-262-262-242-80}$ \\
Mold temperature $\left({ }^{\circ} \mathrm{C}\right)$ & $\underline{110} / 130$ \\
Injection rate $\left(\mathrm{cm}^{3} / \mathrm{s}\right)$ & $\underline{10.6} / 53.1$ \\
Holding pressure $(\mathrm{MPa})$ & $\underline{50 / 60 / 70 / 80 / 90 / 100}$ \\
Holding pressure period $(\mathrm{s})$ & $\underline{\underline{17}}$ \\
Cooling time $(\mathrm{s})$ & $\underline{300}$ \\
Ejector protruding speed $(\mathrm{mm} / \mathrm{s})$ &
\end{tabular}

*Nozzle-metering-compression-feed-under hopper

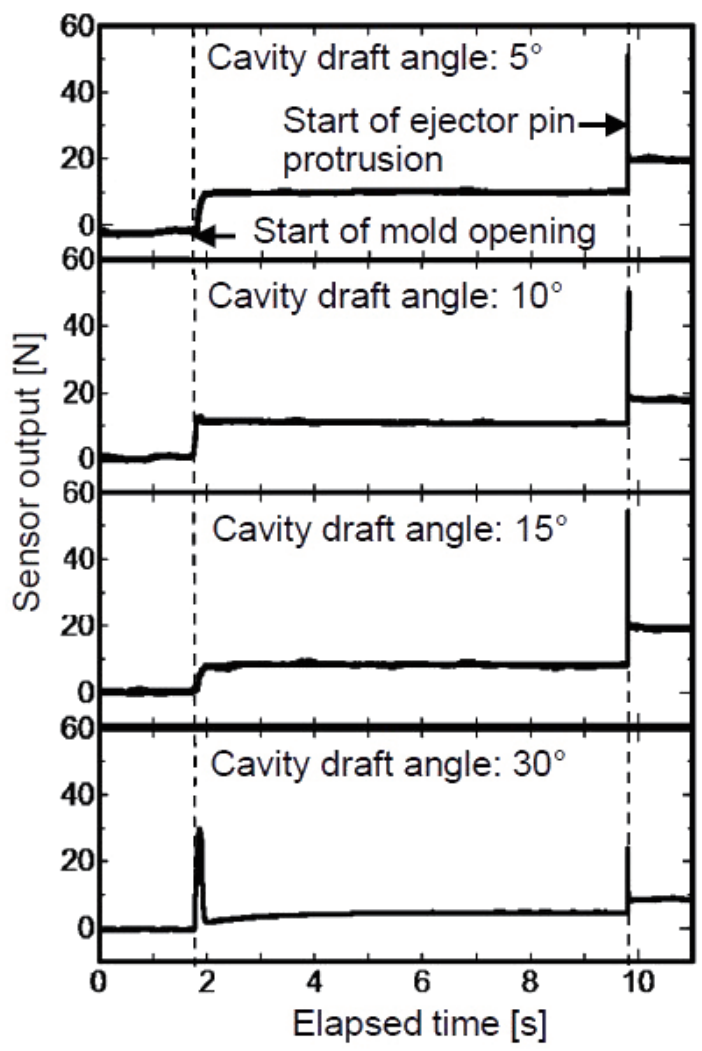

Fig. 8. Demolding resistance measurement results from start of mold opening to start of ejector pin protrusion for various cavity drafts (reference conditions, holding pressure: $60 \mathrm{MPa}$ ).

the start of mold opening regardless of the application of the preload of $10 \mathrm{~N}$. This was caused by the gap generated between the force-receiving surface and sensor support B as a result of (1) the decreasing thickness of the mold upon the application of the mold clamping force to the mold and (2) the deformation of the mold plate subjected to a high pressure from the polymer. At the start of mold opening, the application of mold clamping force to the mold stops and the sensor 
output increases to $10 \mathrm{~N}$. After that, the sensor output becomes constant. Upon the ejection of the molded product, the demolding resistance is recorded as the sensor output. At the cavity draft angle of $30^{\circ}$, a high output of $30 \mathrm{~N}$ is recorded at the start of mold opening. The reason behind this is explained using the free body diagram showing the force acting on the molded product at the start of mold opening, as shown in Fig. 9. Before the start of mold opening, the residual pressure remaining in the molded product during the injection process is applied to the cavity side wall. Therefore, at the start of mold opening, the reaction force $R$ is applied to the surface of the molded product in contact with the cavity side wall. The $x$-direction component of $R x$ is the force that ejects the molded product from the cavity. In contrast, the frictional force $f$ is generated between the molded product and the cavity side wall. The $x$-direction component of $f x$ is the force that prevents the molded product from being ejected. With increasing cavity draft angle $\theta, R x$ increases and $f x$ decreases. At a cavity draft angle of $30^{\circ}, R x$ exceeds $f x$ and the molded product is ejected from the cavity, leading to the pulling of the cavity insert by the molded product. As a result, the sensor output immediately increases. At the same time, the molded product is slightly detached from the cavity wall, leading to a sharp decrease in sensor output. It is also hypothesized that the demolding resistance generated when the molded product is detached from the cavity wall at the stationary mold side upon mold opening is transferred to the cavity insert at the back of the molded product and is measured by the force sensor. To address this problem, for the stationary mold side, we fabricated a special cavity insert that does not generate the demolding resistance and used it for measurement. However, a high sensor output similar to that measured using the conventional cavity insert was recorded at the start of mold opening even when the new special cavity insert was used. Because of this finding, we ruled out the above hypothesis. The mold developed in this study is intended for use during the measurement of the demolding resistance when the molded product is ejected from the cavity, and hence, the generation of demolding resistance during mold opening must be avoided. For this reason, the cavity draft angle should be small.

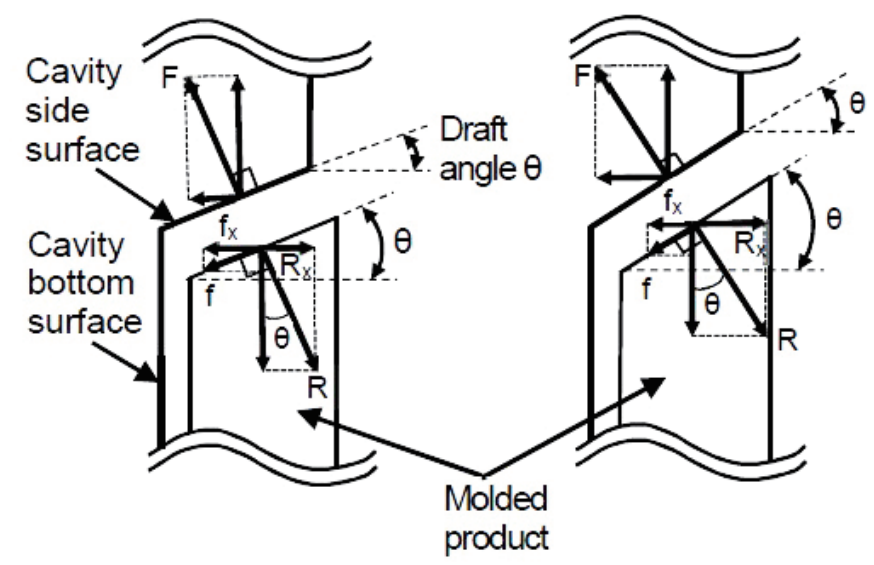

Small draft angle Large draft angle

Fig. 9. Free body diagram of molded product. 


\subsection{Demolding resistance during ejection of molded product}

Figure 10 shows the time courses of the demolding resistance upon the ejection of the molded product and the protruded displacement of ejector pins measured using the displacement sensor (1) at the cavity draft angle of 5, 10, or $15^{\circ}$ under different holding pressures. In the figure, the demolding resistance and the protruded displacement measured at the upper cavity under reference conditions are shown. At the cavity draft angle of $5^{\circ}$ and a holding pressure of less than $80 \mathrm{MPa}$, the demolding resistance sharply increases at the start of ejection and then sharply decreases. In contrast, at a holding pressure of more than $90 \mathrm{MPa}$, the demolding resistance sharply increases at the start of ejection and remains at the same level for some time and then decreases. At the cavity draft angles of 10 and $15^{\circ}$, the demolding resistance sharply increases at the start of ejection and then immediately decreases even at the holding pressure of $90 \mathrm{MPa}$. At the holding pressure of $100 \mathrm{MPa}$, the demolding resistance sharply increases and remains at the same level and then decreases, although the duration of maintaining the high level is shorter in the case of the cavity draft angles of 10 and $15^{\circ}$ than in the case of the cavity draft angle $5^{\circ}$.

The increase in protruded displacement in the duration from $a$ to $b$ at a high holding pressure is slower than that at a low holding pressure. With increasing cavity draft angle, the duration from $a$ to $b$ decreases. In addition, the duration from a sharp increase in demolding resistance to its decrease is in fair agreement with the duration from $a$ to $b$. The reason behind this is explained below. At low holding pressures, $R$ in Fig. 9 decreases regardless of the cavity draft angle because the residual pressure in the molded product is small. As a result, $f x$ decreases and the molded product is smoothly ejected using ejector pins. In contrast, at high holding pressures, $R$ increases. As a result, $f x$ increases, disrupting the operation of the protruding equipment and the ejector plate of the injection molding machine, and the protrusion operation in the duration from $a$ to $b$ in Fig. 10 becomes slow. Therefore, the demolding resistance

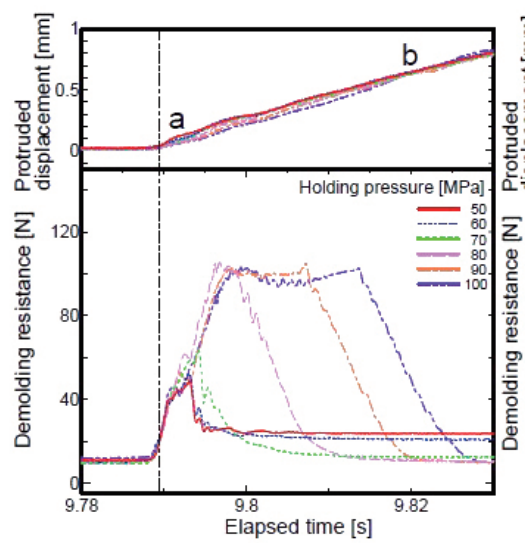

(a)

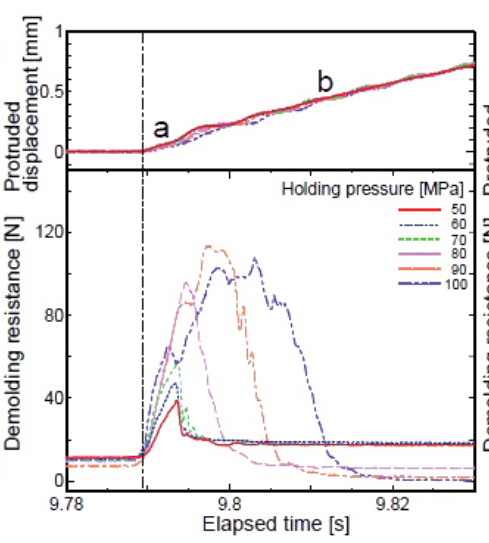

(b)

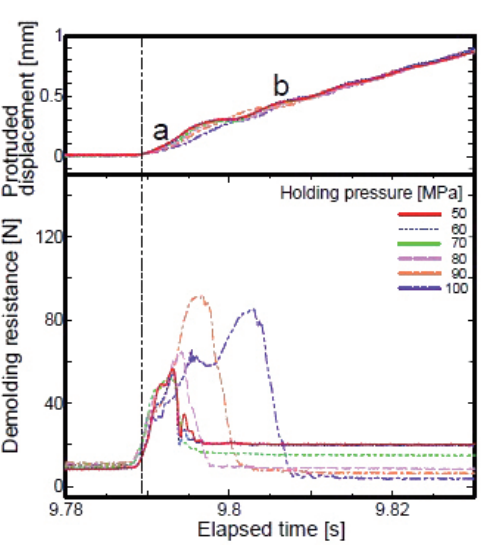

(c)

Fig. 10. (Color online) Demolding resistance and protruded displacement profiles (reference conditions). Cavity draft angles: (a) 5 , (b) 10 , and (c) $15^{\circ}$. 
sharply increases and the high level is maintained for some time. At small cavity draft angles, the above phenomenon is observed at relatively low holding pressures because $f x$ increases with decreasing cavity draft angle. As explained above, with increasing holding pressure and decreasing cavity draft angle, the protrusion operation of the ejector pins becomes slow, affecting the sensor output.

\section{Effect of Molding Conditions on Demolding Resistance}

Using the mold developed in this study, we examined the effects of changes in holding pressure, injection rate, heating cylinder temperature, and mold temperature on the demolding resistance. Figure 11 shows the time courses of the demolding resistance and protruded displacement measured in the upper cavity at the cavity draft angle of $5^{\circ}$ under different molding conditions. When the injection rate is increased, as shown in Fig. 11, the demolding resistance increases under any holding pressure compared with that at a low injection rate (Fig. 10). When the heating cylinder temperature is increased above the reference temperature, as shown in Fig. 10, the demolding resistance increases with increasing heating cylinder temperature at the holding pressures of 60 and $70 \mathrm{MPa}$. When the mold temperature is increased above the reference temperature in Fig. 10, the demolding resistance increases with increasing mold temperature at any holding pressure.

From the output waveform in Fig. 11, the maximum demolding resistance was determined. Figure 12 shows the relationship between the maximum demolding resistance and the holding pressure. The maximum demolding resistance is the average of five measurements and the standard deviation $\sigma$ is also shown in the figure. In this figure, the plots at high holding pressures, at which the operation of the ejector pins is considered to be impaired, are removed. Under any condition, the maximum demolding resistance generally increases with increasing

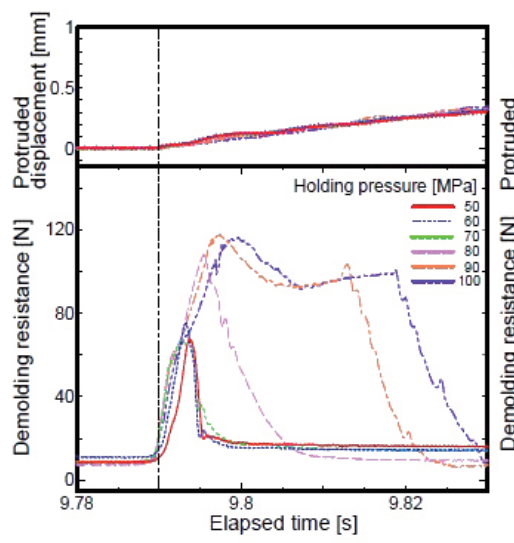

(a)

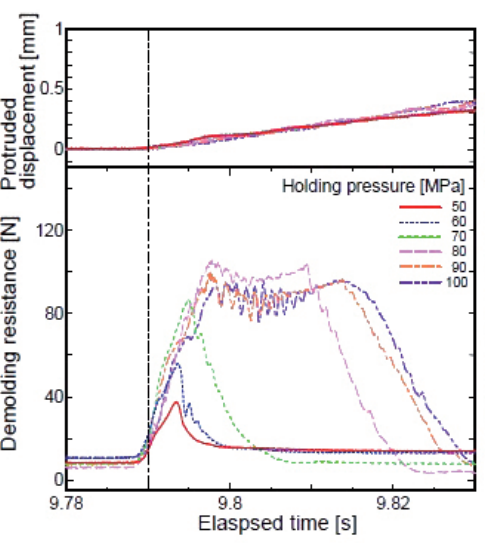

(b)

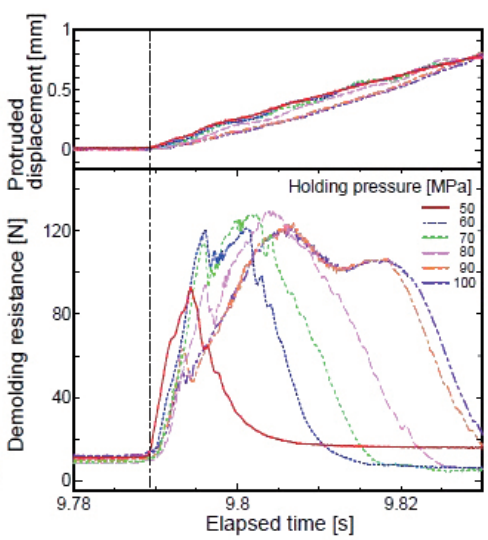

(c)

Fig. 11. (Color online) Demolding resistance measurement results under various molding conditions. Effects of (a) injection rate, (b) heating cylinder temperature, and (c) mold temperature. 


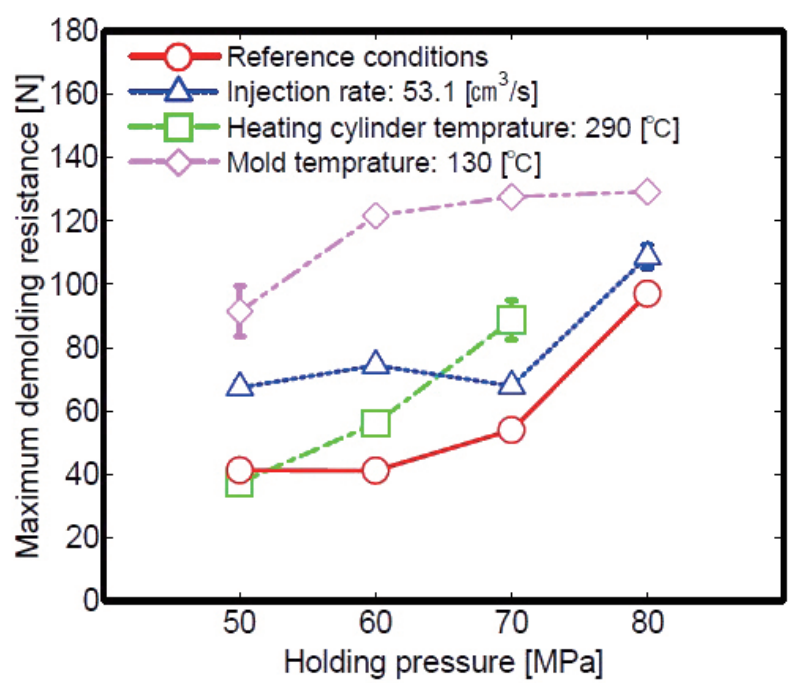

Fig. 12. (Color online) Relationship between maximum demolding resistance and holding pressure under various molding conditions.

holding pressure. In addition, the maximum demolding resistance increases with increasing injection rate, heating cylinder temperature, and mold temperature. In particular, the effects of holding pressure and mold temperature on the maximum demolding resistance were found to be significant.

The reasons behind the above findings are examined below. During the injection process, fountain flow of the polymer toward the cavity wall is induced. Upon contact of the polymer with the cavity wall, the polymer starts to cool and solidify. At high heating cylinder temperature and mold temperature, the temperature of the polymer in contact with the cavity wall is relatively high, leading to a soft polymer with low viscosity in the vicinity of the cavity wall. With increasing injection rate, the polymer is difficult to cool while flowing and shear heating is generated. Therefore, the polymer in the vicinity of the cavity wall is soft with a low viscosity. As explained above, the polymer in the vicinity of the cavity wall becomes soft with a low viscosity with increasing injection rate, heating cylinder temperature, and mold temperature. Therefore, the polymer easily adheres to the cavity wall. In addition, with increasing holding pressure, the force applied to the polymer toward the cavity wall increases. There are many factors that affect the demolding resistance. Among them, the increase in adhesion between the polymer and the cavity wall is considered to be one of the factors in the increase in demolding resistance in this study. The results of our previous study on the measurement of the temperature distribution of a melt flowing in a mold ${ }^{(11)}$ revealed that the temperature in the vicinity of the cavity wall increases with increasing heating cylinder temperature, mold temperature, and injection rate. Furthermore, the results of another previous study on heat-and-cool injection molding ${ }^{(12)}$ demonstrated that the increase in the temperature of a polymer in the vicinity of a cavity wall increases the adhesion between the polymer and the cavity wall. Therefore, our assumption is considered to be valid. 


\section{Conclusions}

We designed and fabricated a mold equipped with high-sensitivity quartz force sensors for demolding resistance measurement and evaluated its performance. Using this mold, we measured the demolding resistance of a fluorine-based polyester lens. We obtained the following conclusions:

(1) A calibration experiment of the sensor output was carried out. The preload applied to the quartz force sensors before the start of mold closing has a significant effect on the sensor output. It was found that the preload should be set at less than $10 \mathrm{~N}$ during measurement.

(2) Using the mold equipped with the high-sensitivity quartz force sensors developed in this study, we succeeded in measuring small demolding resistances of $120 \mathrm{~N}$ or lower for the first time.

(3) The draft angle of the cavity side wall has an effect on the demolding resistance. Namely, at large draft angles, the molded product is slightly detached from the cavity wall as a result of the force originating from the residual pressure in the molded product, leading to a change in sensor output. In contrast, at small draft angles, the protrusion operation of the ejector pins becomes slow at high holding pressures, leading to a change in sensor output.

(4) It was clarified that the maximum demolding resistance increases with increasing holding pressure, injection rate, heating cylinder temperature, and mold temperature.

As explained above, we clarified the characteristics of the mold developed in this study and demonstrated its advantages. In the future, studies on the demolding resistance under various conditions, i.e., various types of polymer and surface properties and surface treatment conditions of the cavity, will be carried out.

\section{Acknowledgments}

We are grateful to Kistler Japan Co., Ltd., for kindly lending us the high-sensitivity quartz force sensors and Ikegami Mold Engineering Co., Ltd., for cooperation in the design and fabrication of the mold used in this study.

\section{References}

1 F. Tsuchiya and H. Suzuki: J. Surf. Finish. Soc. Jpn. 58 (2007) 56.

2 TS Nanocoat Corp.: http://www.nanocoat-ts.com (accessed January 2019).

3 M. Fujiwara, K. Suzuki, Y. Miura, H. Sasaki, Y. Sasaki, H. Chiba, S. Satou, M. Kasuya, and T. Ono: Preprints of Seikei-Kakou Autumnal Meeting 2011 (2011) 159.

4 Plastron Corp.: http://www.plastron.co.jp (accessed January 2019).

5 Fuji Manufacturing Co., Ltd.: http://www.fujimfg.co.jp/english/index.html (accessed January 2019).

6 Y. Kobayashi, K. Shirai, and T. Sasaki: J. Jpn. Soc. Precis. Eng. 67 (2001) 510.

7 H. Yamamoto, Y. Ohkubo, K. Ogawa, and K. Utsumi: J. Jpn. Soc. Polym. Process. 21 (2009) 38.

8 K. Oota, W. Natsu, and M. Okabe: Int. J. Jpn. Soc. Precis. Eng. 84 (2018) 473.

9 G. R. Berger, W. Friesenbichler, G. Schofer, and G. Freudenschuss: Proc. Polym. Process. Soc. 24th Annu. Meeting (2008) 382.

10 H. Yokoi and T. Shito: Proc. Polym. Process. Soc. 24th Annu. Meeting (2008) 294.

11 Y. Murata: J. Jpn. Soc. Polym. Process. 23 (2011) 700.

12 Y. Murata and M. Kuramochi: Int. J. Automation Tech. 10 (2016) 79. 